\title{
BEAM-BASED MEASUREMENTS OF PERSISTENT CURRENT DECAY IN RHIC**
}

\author{
W. Fischer, A. Jain and S. Tepikian, Brookhaven National Lab, Upton, NY 11973, USA
}

\section{Abstract}

The two RHIC rings are equipped with superconducting dipole magnets. At injection, induced persistent currents in these magnets lead to a sextupole component. As the persistent currents decay with time, the horizontal and vertical chromaticities change. From magnet measurements of persistent current decays, chromaticity changes in the machine are estimated and compared with chromaticity measurements.

\section{INTRODUCTION}

Persistent currents are eddy currents that are induced in the filaments of superconducting magnets through a change in the magnetic field. They generate all multipoles which are allowed by coil symmetry, i.e. the normal multipoles $b_{0}, b_{2}, b_{4}, \ldots$ in dipoles and $b_{1}, b_{5}, b_{9}, \ldots$ in quadrupoles. At a constant field during injection these currents decay with time. Decay rates typically vary from magnet to magnet and depend on the magnetic field history as well as on cable properties [1].

At injection energy, the sextupole field errors $b_{2}$ from persistent currents can be significant due to their size and time-dependence. Their uncorrected change during injection may be unacceptable for operation. An overview of various hadron colliders can be found in Ref. [2]. Time dependence is not only observable as a slow decay at the injection level but also as a snap-back to the original multipole value when acceleration starts. For an effective correction of persistent current effects, reproducibility and predictability are important. In this article we compare for both RHIC rings the time-dependent chromaticity changes expected from magnet measurements with measured chromaticity changes.

During the year 2000 gold run, beam was injected in RHIC at a dipole current of $462 \mathrm{~A}$. Time-dependent chromaticity measurements in both the Blue and Yellow ring started 2 min after reaching the injection current and extended over $15 \mathrm{~min}$.

\section{MAGNET MEASUREMENTS OF PERSISTENT CURRENT DECAY}

The expected time-dependent chromaticity change can be deduced from two types of magnet measurements. First, the persistent current decay was measured in 20 magnets at $660 \mathrm{~A}$, a current higher than the injection current of

\footnotetext{
* Work performed under the auspices of the US department of Energy. Copyright The American Physical Society 2001. All rights reserved. Except as provided under U.S. copyright law, this work may not be reproduced, resold, distributed or modified without the express permission of the The American Physical Society. The archival version of this work was published in Phys. Rev. ST Accel. Beams 4, 041002 (2001).
}

Table 1: Change in $b_{2}$ is computed from $2 \mathrm{~min}$ to $17 \mathrm{~min}$ with the fit functions (1) and (2).

\begin{tabular}{lc|c}
\hline \hline Measurement & $\begin{array}{c}\text { Logarithmic } \\
\Delta b_{2}\left[10^{-4}\right]\end{array}$ & $\begin{array}{c}2 \text { exponentials } \\
\Delta b_{2}\left[10^{-4}\right]\end{array}$ \\
\hline 20 magnets 660A, average & $0.49 \pm 0.15$ & $0.39 \pm 0.14$ \\
20 magnets scaled to 462A & $0.70 \pm 0.22$ & $0.56 \pm 0.20$ \\
1 magnet at 470A & $0.83 \pm 0.07$ & $0.39 \pm 0.02$ \\
\hline \hline
\end{tabular}

462 A. Second, the decay was also measured in a single dipole magnet at $470 \mathrm{~A}$. Ideally, the persistent current decay in magnets should be measured under the same conditions as in machine operation. However, the $20 \mathrm{RHIC}$ magnets were measured during production assuming an injection current higher than the one finally used in operation.

The decaying sextupole components were fitted to two functions. First, a logarithmic time dependence was assumed according to

$$
b_{2}=c_{0}+c_{1} \log _{10}\left(t / \tau_{1}\right) \text { with } \tau_{1}=1 \mathrm{~s}
$$

Such a time-dependence is characteristic of a relaxation process, namely the flux creep phenomenon in hard superconductors. The HERA magnets could be characterized this way [1]. Another fit for the RHIC magnets can be obtained with two superimposed exponentially decaying terms according to

$$
b_{2}=c_{0}+c_{1} e^{-t / \tau_{1}}+c_{2} e^{-t / \tau_{2}} .
$$

An exponential decay is expected when the eddy current loops are partly superconductive and partly resistive.

For the measurement of the 20 magnets Tab. 1 shows the change in $b_{2}$, computed with both fit functions over $15 \mathrm{~min}$ starting $2 \mathrm{~min}$ after reaching $660 \mathrm{~A}$. The $\Delta b_{2}$ errors are the square root of the average quadratic deviation between measured and fitted values. The fitted functions are also shown in Fig. 1. The fit with Eq. (1) overestimates the decay while the fit (2) underestimates the decay.

Since the persistent currents are approximately independent of the main field at transport currents well below the critical current, the coefficients $c_{0}, c_{1}$ and $c_{2}$ in Eqs. (1) and (2) may be scaled with the reference field. The result of scaling is also shown in Tab. 1. The simple scaling is, however, only approximate and the exact scaling is not known. A more detailed'discussion of scaling can be found in Ref. [2].

The result of the single magnet measurement at 470 A [3] are shown in Fig. 2 and in Tab. 1. It is evident that a simple linear relation between $b_{2}$ and $\log (t)$ is not followed by the data over the entire range.

The expected change in $b_{2}$ from the scaled measurements of the 20 magnets at $660 \mathrm{~A}$ differs from the expected change in $b_{2}$ from the single magnet measurement at $470 \mathrm{~A}$ 


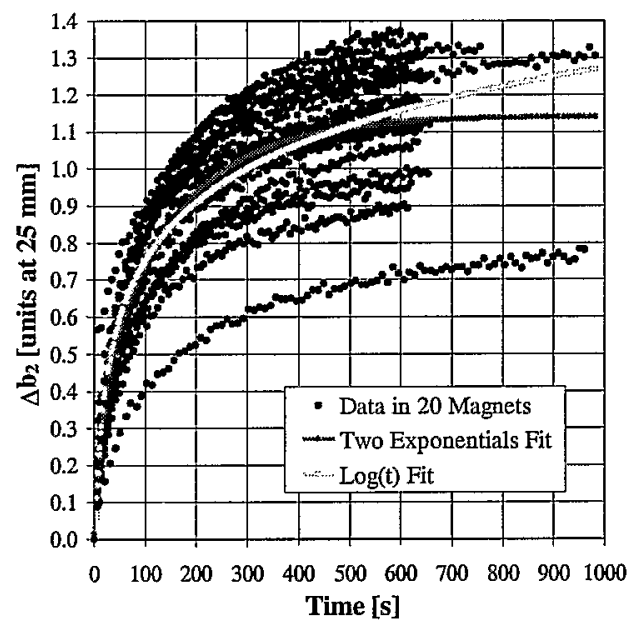

Figure 1: Time-dependent change of the sextupole coefficient in 20 RHIC dipoles at $660 \mathrm{~A}$.

by some $25 \%$, with error intervals overlapping (see Tab. 1 ). However, as Fig. 1 shows, individual magnets can differ in their behavior significantly from each other.

A discussion of the magnetic cycle and the ramp rate on the persistent current decay as well as references to magnet measurements of persistent current decays for the Tevatron and HERA can be found in Ref. [2].

\section{EXPECTED CHROMATICITY CHANGE FROM PERSISTENT CURRENT DECAY}

The change in the chromaticity $\xi_{x, y}=\Delta Q_{x, y} /(\Delta p / p)$ associated with the change of the sextupole component $\Delta b_{2}$ can be computed as [4]

$$
\Delta \xi_{x, y}= \pm \frac{1}{2 \pi} \frac{1}{(B \rho)} \oint \beta_{x, y}(s) \frac{B_{r e f} \Delta b_{2}(s)}{r_{0}^{2}} \eta_{x}(s) \mathrm{d} s .
$$

where $\beta_{x, y}$ is the lattice beta function and $\eta_{x}$ the dispersion. $B_{\text {ref }}$ may be different from the $B$ in the rigidity $(B \rho)$. Assuming sextupole errors only in dipoles Eq. (3) can be approximated as

$$
\Delta \xi_{x, y}= \pm \frac{1}{2 \pi} \frac{1}{(B \rho)} \frac{B_{r e f} \Delta b_{2}}{r_{0}^{2}} N I_{x, y}
$$

where $N$ denotes the number of dipoles and the integrals

$$
I_{x, y}=\int_{l_{1}}^{l_{2}} \beta_{x, y}(s) \eta_{x}(s) \mathrm{d} s
$$

extend over a dipole in a FODO cell. In Eq. (4) we use only the average change in the sextupole coefficient $\Delta b_{2}$. Furthermore, we only consider dipoles in FODO cells since contributions from the interaction regions are small [2]. The integrals $I_{x, y}$ can be computed using a thin-lens approximation for the magnets in the cell $[2,5]$. The result of a numeric integration using the RHIC cell parameter [6] is shown in Tab. 2 (analytical).

The expected chromaticiy change can also be computed with optics programs. For RHIC we use MAD. and Teapot. Both programs can introduce magnetic field errors in the

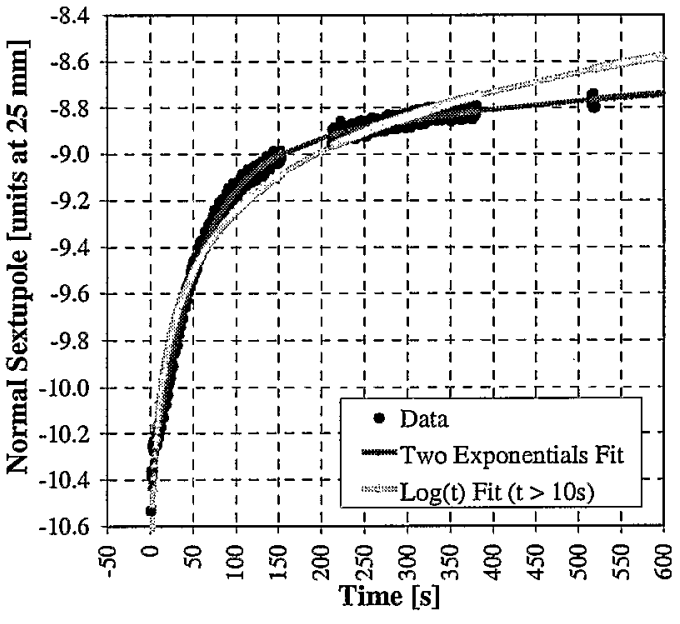

Figure 2: Time-dependent sextupole coefficient in a single RHIC dipole at 470A.

Table 2: The effect of a change in the sextupole component on the chromaticity. $b_{2}$ is given in units of $10^{-4}$ at a reference radius of $r_{0}=25 \mathrm{~mm}$.

\begin{tabular}{lcccc}
\hline \hline & \multicolumn{2}{c}{ Natural } & \multicolumn{2}{c}{ Chromaticity due } \\
& \multicolumn{2}{c}{ Chromaticity } & \multicolumn{2}{c}{ to $b_{2}$ in dipoles } \\
& $\xi_{x, n}$ & $\xi_{y, n}$ & $\xi_{x, b_{2}}$ & $\xi_{y, b_{2}}$ \\
\hline Analytical & - & - & $+4.9 \times b_{2}$ & $-4.4 \times b_{2}$ \\
MAD & -54.9 & -56.5 & $+4.9 \times b_{2}$ & $-4.3 \times b_{2}$ \\
Teapot & -54.7 & -56.6 & $+4.2 \times b_{2}$ & $-4.0 \times b_{2}$ \\
\hline \hline
\end{tabular}

lattice. The results for both programs are also shown in Tab. 2. The analytical estimate and the coefficients determined from MAD agree very well. The Teapot coefficients are slightly different since Teapot uses a single kick approximation for every dipole while the analytical estimate and MAD use a thick lens model.

\section{MEASUREMENTS OF TIME-DEPENDENT CHROMATICITY}

The chromaticity was determined by measuring the tune at different average radii and therefore momenta. The tune was obtained from the Fourier transforms of transverse beam oscillations over 512 turns after the beam was excited with a single small kick [7]. The momentum was changed by $\Delta p / p= \pm 0.0017$. The chromaticity was measured every 16 seconds beginning 2 minutes after reaching the injection plateau. Measurements extended over 15 minutes. Both the Blue and the Yellow ring were measured in gold operation. In Fig. 3 the results of the time-dependent chromaticity measurements are shown. With MAD it was determined that tune changes due to second order chromaticy coefficients can be disregarded.

We fit the chromaticity to two functions equivalent to Eq. (1) and Eq. (2). However, the experimental data make a fit to five parameters like in Eq. (2) difficult. Furthermore the fast decaying component has largely decayed when the beam-based measurements start. We therefore set $\bar{c}_{1}=0$ and perform only a three parameter fit. In addition, we force the time constant $\bar{\tau}_{2}$ to be the same in the Blue and Yellow rings. 


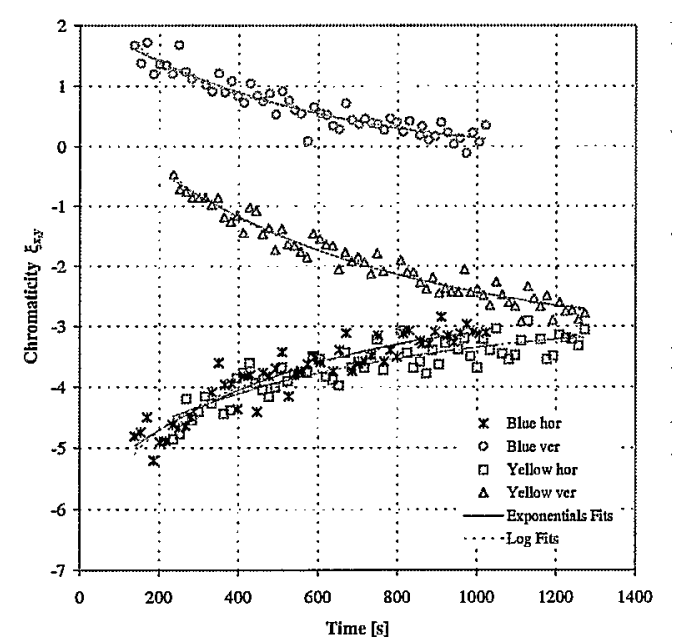

Figure 3: Measured chromaticity in the two RHIC rings on a linear time scale.

The change in $\xi_{x, y}$ computed from $2 \mathrm{~min}$ to $17 \mathrm{~min}$ with the fitted functions is shown in Tab. 3 along with the expectations from the magnet measurements. The beambased measurements agree better with the single magnet measurement than with the scaled measurement of the 20 magnets. Generally, a better agreement is obtained for the exponential fits. For these, the scaled $660 \mathrm{~A}$ measurements show an average deviation of $-28 \%$. For the single $470 \mathrm{~A}$ measurement the average is $4 \%$. Fig. 4 also shows the comparison between the expectation from both magnet measurements and the chromaticity measurements.

\section{SUMMARY}

Magnet measurements of persistent current decays in RHIC dipoles show neither a clear logarithmic nor a clear exponential dependence but indicate both components. The relatively small chromaticity changes that can be computed from the measurements of a single magnet agree well with beam-based measurements.

A single magnet was found to be a good proxy for both the Blue and Yellow ring with respect to the persistent current decay at injection. However, since decay times vary considerably from magnet to magnet, predictions of the chromaticity change should not rely on measurements of

Table 3: Change in $\xi_{x, y}$ computed from $2 \mathrm{~min}$ to $17 \mathrm{~min}$ with the fitted functions. The $\Delta \xi_{x, y}$ errors correspond to the square root of the average quadratic deviation between measured and fitted values.

\begin{tabular}{lc|c}
\hline Measurement & $\begin{array}{c}\text { Logarithmic } \\
\Delta \xi_{x, y}[1]\end{array}$ & $\begin{array}{c}\text { 2 Exponentials } \\
\Delta \xi_{x, y}[1]\end{array}$ \\
\hline \hline Horizontal & & \\
20 magnets, 660 A scaled & $3.45 \pm 1.06$ & $2.73 \pm 0.97$ \\
single magnet, 470 A & $4.05 \pm 0.34$ & $1.89 \pm 0.10$ \\
\hline Blue ring & $2.16 \pm 0.22$ & $1.98 \pm 0.21$ \\
Yellow ring & $1.73 \pm 0.20$ & $1.48 \pm 0.21$ \\
\hline \hline Vertical & & \\
20 magnets, 660 A scaled & $-3.10 \pm 0.95$ & $-2.45 \pm 0.87$ \\
single magnet, 470 A & $-3.64 \pm 0.31$ & $-1.70 \pm 0.09$ \\
\hline Blue ring & $-1.68 \pm 0.16$ & $-1.54 \pm 0.15$ \\
Yellow ring & $-2.85 \pm 0.15$ & $-2.44 \pm 0.15$ \\
\hline \hline
\end{tabular}

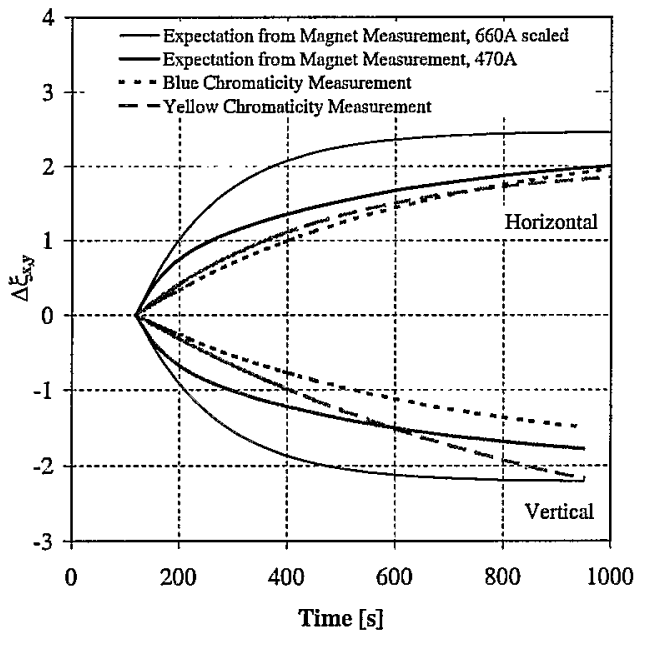

Figure 4: Comparison between chromaticity changes expected from magnet measurements and measured chromaticity changes. In all cases the exponential fit functions are shown.

a single magnet.

The agreement with a scaled measurement of $20 \mathrm{mag}-$ nets, carried out at a higher current and with a different cycle, is less satisfactory. This suggests that the simple scaling law used is only a crude approximation. For machines, for which a detailed knowledge of the persistent current behavior is essential for operation, magnet measurements should therefore be done under conditions as close as possible to those encountered in machine operation.

\section{ACKNOWLEDGMENTS}

We are thankful to R. Thomas and W. Louie for help with the magnet measurements and to the RHIC operations teams for help during the beam based measurements. We are grateful to A. Drees for support of the tune measurement systems. A special thanks to T. Satogata and J. van Zeijts who supported this study in many ways.

\section{REFERENCES}

[1] P. Schmïser, "Cos $\theta$ Superconducting Magnets", in "Handbook of Accelerator Physics and Engineering" edited by A.W. Chao and M. Tigner, World Scientific (1999).

[2] W. Fischer, A. Jain and S. Tepikian, "Beam-based Measurements of Persistent Current Decay in the Relativistic Heavy Ion Collider", Phys. Rev. ST Accel. Beams 4, 041002 (2001).

[3] A. Jain et al., "Time Decay, Snap-back and Ramp Rate Effects in RHIC $8 \mathrm{~cm}$ Dipoles and Quadrupoles", BNL Magnet Division Note 593-11 (AM-MD-294) (2000).

[4] M. Conte, W.W. MacKay, "An Introduction to the Physics of Particle Accelerators", World Scientific (1991).

[5] E. Keil, "Lattices for Collider Storage Rings", in "Handbook of Accelerator Physics and Engineering" edited by A.W. Chao and M. Tigner, World Scientific (1999).

[6] H. Hahn (editor) "RHIC Design Manual", revision of October 2000.

[7] P. Cameron, R. Connolly, A. Drees, T. Ryan, H. Schmickler, T. Shea, and D. Trbojevic, "ARTUS: A Rhic TUne monitor System", BNL RHIC/AP/156 (1998). 